\title{
A novel electrochemical method for efficient reduction of disulfide bonds in peptides and proteins prior to MS detection
}

\author{
Agnieszka Kraj • Hendrik-Jan Brouwer • \\ Nico Reinhoud • Jean-Pierre Chervet
}

Received: 8 August 2013 / Revised: 10 September 2013 / Accepted: 13 September 2013 /Published online: 29 September 2013

(C) The Author(s) 2013. This article is published with open access at Springerlink.com

\begin{abstract}
A novel electrochemical (EC) method for fast and efficient reduction of the disulfide bonds in proteins and peptides is presented. The method does not use any chemical agents and is purely instrumental. To demonstrate the performance of the EC reactor cell online with electrospray mass spectrometry, insulin and somatostatin were used as model compounds. Efficient reduction is achieved in continuous infusion mode using an EC reactor cell with a titaniumbased working electrode. Under optimized conditions, the presented method shows almost complete reduction of insulin and somatostatin. The method does not require any special sample preparation, and the EC reactor cell makes it suitable for automation. Online EC reduction followed by collisioninduced dissociation fragmentation of somatostatin showed more backbone cleavages and improved sequence coverage. By adjusting the settings, the EC reaction efficiency was gradually changed from partial to full disulfide bonds reduction in $\alpha$-lactalbumin, and the expected shift in charge state distribution has been demonstrated. The reduction can be controlled by adjusting the square-wave pulse, flow rate or mobile phase composition. We have shown the successful use of an EC reactor cell for fast and efficient reduction of disulfide bonds for online mass spectrometry of proteins and peptides. The possibility of online and gradual disulfide bond reduction adds a unique dimension to characterization of disulfide bonds in midand top-down proteomics applications.
\end{abstract}

Electronic supplementary material The online version of this article (doi:10.1007/s00216-013-7374-3) contains supplementary material, which is available to authorized users.

A. Kraj $(\bowtie) \cdot$ H.-J. Brouwer • N. Reinhoud · J.-P. Chervet Antec, Industrieweg 12, 2382NV Zoeterwoude, The Netherlands e-mail: agnieszka.kraj@myAntec.com
Keywords Electrochemistry $\cdot$ Mass spectrometry $\cdot$ Disulfide bond reduction $\cdot$ Square-wave pulse $\cdot$ Proteomics

\section{Introduction}

Disulfide bonds are some of the most important posttranslational modifications of proteins as they stabilize the native conformation of a protein and regulate the protein's biological function [1]. The determination of the arrangement of the disulfide bonds is crucial for understanding the folding processes of a protein.

Disulfide bond reduction is furthermore a prime requisite for protein identification by mass spectrometry (MS). Any "bottom-up" protocol for identification and sequencing of a protein involves disulfide bond cleavage prior to enzymatic cleavage $[2,3]$. In "top-down" approaches, sequence information is obtained from mass spectrometric fragmentation of high-mass multiply charged ions [4]. As proteins with disulfide bonds show resistance to fragmentation, disulfide bond reduction is still necessary for comprehensive sequence analysis by low-energy collision-induced dissociation (CID) $[5,6]$. Recently, alternative mass spectrometric-based fragmentation techniques such as electron capture dissociation [7] and electron transfer dissociation [8] have been used to cleave the disulfide bonds and the peptide backbone [8-10]. Furthermore, due to the fact that $\mathrm{N}-\mathrm{C} \alpha$ backbone cleavage can be competing with disulfide bond reduction, even for sequencing with the electron capture dissociation and electron transfer dissociation cleavage of disulfide bonds is required [10].

The common approach for disulfide bond cleavage is based on chemical reduction using agents such as dithiothreitol [11] or thiol-free reducing agents as tris(2-carboxyethyl)phosphine (TCEP) [12] followed by alkylation of free sulfhydryl groups to prevent their spontaneous oxidation and proteolytic 
digestion (e.g. with trypsin) prior to mass spectrometric analysis [2,3]. Despite considerable success, this strategy has some limitations. It is usually considered to be timeconsuming, and can hardly be adapted for online MS.

In seeking to establish a more advanced method, electrochemistry (EC) has been introduced recently. EC is a purely instrumental approach that can be online hyphenated to MS. EC combined offline and online with mass spectrometry has been successfully used for the investigation of drug metabolism [13], environmental behaviour of xenobiotics [14] but also nucleic acid oxidation [15]. Application of a square wave pulse potential has been described for modulating the electrochemical reaction products [16]. Added value of electrochemistry has also been noticed in protein research [17]. Recently, a paper on the electrochemical oxidation and the oxidative cleavage of tyrosine and tryptophan containing peptides has been published by Roeser et al. [18].

The first report regarding electrochemical reduction of disulfide bonds of proteins was published in 1964 by Cecil et al. [19], and additional reports followed [20, 21]. Disulfidecontaining peptides were determined by means of highperformance liquid chromatography (HPLC) with electrochemical detection (ECD) [22] and by HPLC/ECD and mass spectrometry $[23,24]$. Although ECD can detect disulfides at very low levels (e.g. the limit of detection for Vasopressin and Oxytocin was 1 pmol on column [22]), chromatographic fractions had to be collected and sample preparation was required before MS analysis could be conducted [23, 24]. However, the first mass spectrometric analysis of electrochemically cleaved disulfide bonds was accomplished by Girault and co-workers in 2008 [25]. They observed reduction of disulfide bonds during matrix-assisted laser desorption ionization (MALDI) of peptides from targets modified with titanium dioxide nanoparticles. Their method required an addition of glucose, a non-volatile electron donor to the protein before the deposition on the MALDI plate. Chen and coworkers pioneered the use of online EC/MS for reduction of disulfide bonds in peptide and protein. Amalgam [26] and conductive diamond [27-29] was employed as electrode material for disulfide reduction. Mass spectrometric analysis was enabled by desorption electrospray ionization. Although reduction on conductive diamond electrode substantially increased backbone cleavage by different MS fragmentation techniques, the reduction efficiency for proteins was much lower than the reduction by DTT [Fig. S1, see Electronic supplementary material (ESM)] [29]. Our optimized protocol provides not only stable but also very efficient (close to $100 \%$ ) reduction of disulfide bonds as well in peptides as in proteins. Furthermore, our method using the electrochemical reactor cell is well suitable for automated online LC/EC/MS or EC/LC/MS applications.

Herein, we present an alternative, electrochemical method for the fast and efficient reduction of disulfide bonds in peptides and proteins. The unique properties of an EC reactor cell with a titanium-based working electrode have been investigated and a special square-wave potential pulse was developed. A complete or near to complete reduction of the disulfide bonds of the tested substances has been demonstrated.

\section{Experimental}

\section{Chemicals}

Insulin from bovine pancreas, $\alpha$-lactalbumin from bovine milk and formic acid (99\%) were obtained from Sigma Aldrich (The Netherlands). Somatostatin 14 was purchased from Bachem (Switzerland). Acetonitrile (99.9 \%) was obtained from Acros organics (Belgium). All these reagents were used as received without further purification. Deionized water $(18.2 \mathrm{M} \Omega . \mathrm{cm})$ used for all experiments was obtained from a Barnstead Easypure II system (Thermo Fisher Scientific, USA).

\section{EC/MS of peptides and proteins}

All experiments were performed on a ROXY EC system (Antec, The Netherlands) consisting of a ROXY Potentiostat, equipped with an electrochemical reactor cell ( $\mu$-PrepCell, Antec, The Netherlands) and an infusion pump (Harvard Apparatus, USA). The Roxy system was online hyphenated to a LTQ-FT mass spectrometer (Thermo Fisher Scientific, USA). A schematic drawing of the instrumental set-up used is presented in Fig. 1.

Electrochemical disulfide bond reduction was performed in the $\mu$-PrepCell. This thin-layer electrochemical reactor cell consisted of a titanium-based working electrode (WE) specifically optimized for efficient reduction [30], a titanium auxiliary (counter) electrode (AUX) and a $\mathrm{Pd} / \mathrm{H}_{2}$ reference electrode (REF). A $150-\mu \mathrm{m}$ spacer was used to separate the WE and the auxiliary electrode inlet block giving a cell volume of approximately $11 \mu \mathrm{L}$. The ROXY EC system was controlled by Dialogue software (Antec, The Netherlands). An electrical grounding union was used to decouple the electrochemical from the ESI high voltage. The connections were made of PEEK tubing $(127 \mu \mathrm{m}$ i.d.). Details of the square-wave pulses used to accomplish disulfide reduction are shown in Fig. 2. Aqueous solutions of $0.9-5-\mu \mathrm{mol} / \mathrm{L}$ solutions of the insulin $(900 \mathrm{nmol} / \mathrm{L})$, somatostatin $(3 \mu \mathrm{mol} / \mathrm{L})$ and $\alpha$-lactalbumin $(5 \mu \mathrm{mol} / \mathrm{L})$ containing $1 \%$ formic acid and $10-50 \% \mathrm{ACN}$ were used for EC/MS experiments. Sample solutions were delivered to the electrochemical cell by the integrated infusion pump. The flow rate was set to $50 \mu \mathrm{L} / \mathrm{min}$. For tuning the reduction efficiency flow rates up to $200 \mu \mathrm{l} /$ min were applied. The electrochemical cell was thermostated to $35^{\circ} \mathrm{C}$. 


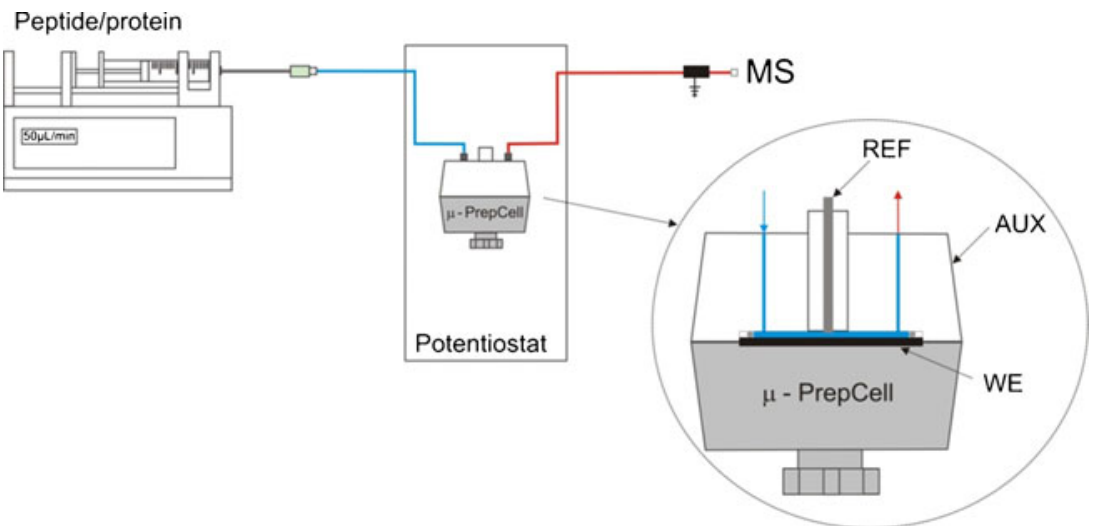

Fig. 1 Schematics of ROXY EC/MS setup for direct infusion experiments. Shown are: the infusion pump containing the syringe with a protein/peptide sample, the $\mu$-PrepCell placed in the ROXY potentiostat and the grounding union between the EC reactor cell outlet and ESI inlet. The blue line corresponds to $45 \mathrm{~cm}(0.254 \mathrm{~mm}$ id $)$ of PEEK tubing. The

Mass spectrometric experiments were performed on an LTQ-FT mass spectrometer (Thermo Fisher Scientific, USA). ESI-MS was performed in positive ion mode. For each peptide/protein, optimization of mass spectrometric conditions was accomplished by infusion of the native form. The spray voltage ranged between 3.2 and $3.6 \mathrm{kV}$. The sheath gas was set to $15-20$ arbitrary units (AU), and the auxiliary gas was set $0-5$ AU. Tube lens voltages of $90-200 \mathrm{~V}$ were used. The transfer capillary temperature was set to $275^{\circ} \mathrm{C}$. MS spectra were recorded from 100 to $2,000 \mathrm{~m} / \mathrm{z}$ with the FT analyzer at a resolution of 100,000 . For selected optimization experiments only LTQ analyzer was used to register MS spectra. MS/MS spectra were generated by CID in the ion trap. Normalized collision energies of $15-35 \%$ were used to initiate fragmentation. MS/MS spectra were acquired with the LTQ analyzer. Accumulation times varied from $100 \mathrm{~ms}$ for the LTQ to $500 \mathrm{~ms}$ for FT. Mass spectra were recorded with Xcalibur software (version 2.0.7, Thermo Fischer Scientific, USA).

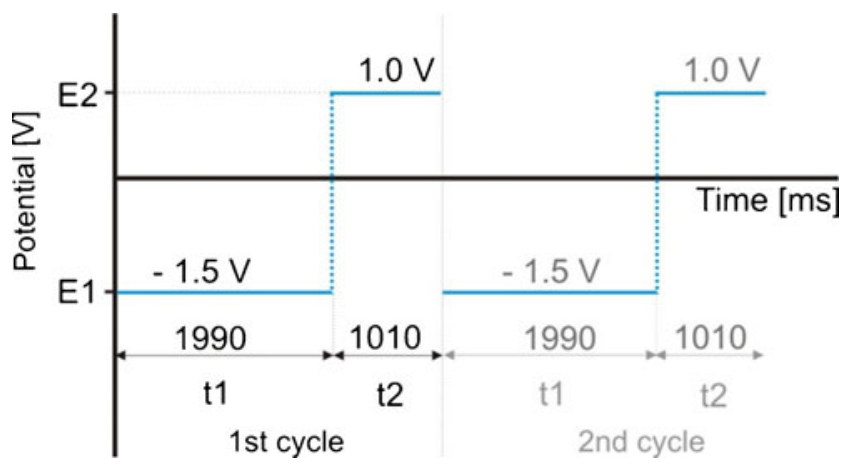

Fig. 2 A schematic representation of the square-wave pulse. Under optimized conditions, the potentials were $-1.5 \mathrm{~V}$ (E1) and $+1.0 \mathrm{~V}$ (E2) and time intervals were $1,990 \mathrm{~ms}(\mathrm{t} 1)$ and $1,010 \mathrm{~ms}(\mathrm{t} 2)$, unless specified otherwise red line corresponds to $100 \mathrm{~cm}(0.127 \mathrm{~mm}$ i.d.) of outlet PEEK tubing. In the insert, a more detailed schematic of $\mu$-PrepCell with a working (WE), auxiliary (AUX) and reference (REF) electrode is shown. A blue and red arrow corresponds to the reactor inlet and outlet, respectively. A fluidic path inside the cell is represented as a blue line

\section{Results}

\section{EC/MS of insulin}

Insulin consists of 51 amino acids forming two chains, A and $\mathrm{B}$, and contains 3 disulfide bonds. Two inter-chain disulfide bonds connect chain $\mathrm{A}$ and $\mathrm{B}$ and one intra-chain disulfide bond is located on chain A (Fig. 3).

Disulfide bond reduction cleaves the molecule. Thus, chain $\mathrm{A}$ and $\mathrm{B}$ are unequivocally distinguishable from the native form by MS. Accordingly, insulin is ideally suited as model compound to characterize the efficiency of electrochemical disulfide bond reduction. The reduction efficiency can be easily established by measuring the percent disappearance of the substrate and appearance of product. For further optimization experiments, appearance of chain B was chosen as an insulin reduction marker.

Efficiency was calculated as ratio between signal abundance of insulin with no potential applied (Cell $\mathrm{OFF}$, no reduction) and with a square-wave pulse applied (Cell $\mathrm{ON}_{\mathrm{N}}$, reduction) according the following equation: $100 \% \times\left(\mathrm{Cell}_{\mathrm{OFF}}-\mathrm{Cell}_{\mathrm{ON}}\right) /$ Cell $_{\mathrm{OFF}}$. Where Cell $\mathrm{OFF}_{\text {and }}$ Cell $\mathrm{ON}$ correspond to the abundance of the average mass spectra from the plateau region measured in the narrow selected ion monitoring mode (mass range of 1,140-1,170). The +5 -fold protonated ion of intact insulin observed at $\mathrm{m} / \mathrm{z} 1,147$ was chosen to calculate the efficiency.

The mass spectrum of the native form of insulin is shown in Fig. 4a. $[\mathrm{M}+4 \mathrm{H}]^{4+},[\mathrm{M}+5 \mathrm{H}]^{5+},[\mathrm{M}+6 \mathrm{H}]^{6+}$ ions were observed. The mass spectrum obtained after online electrochemical reduction is depicted in Fig. 4b. As expected, electrochemical disulfide bond reduction gave rise to cleavage of insulin. Nevertheless, mainly ions corresponding to chain B were detected as $[\mathrm{M}+3 \mathrm{H}]^{3+},[\mathrm{M}+4 \mathrm{H}]^{4+},[\mathrm{M}+5 \mathrm{H}]^{5+}$ (Fig. 4b). Chain A contains multiple acidic residues (glutamic acid at position 
Fig. 3 Sequence of somatostatin, bovine insulin and $\alpha$-lactalbumin. Positions of disulfide bonds are indicated

Fig. 4 Mass spectra of $0.9 \mu \mathrm{mol} /$ $\mathrm{L}$ insulin in $1 \%$ formic acid and $10 \% \mathrm{ACN}$ with EC reactor cell OFF (a) and ON using a Ti-based working electrode (b). For pulse settings see Fig. 2
Somatostatin AGCKNFFWKTFTSC

Insulin

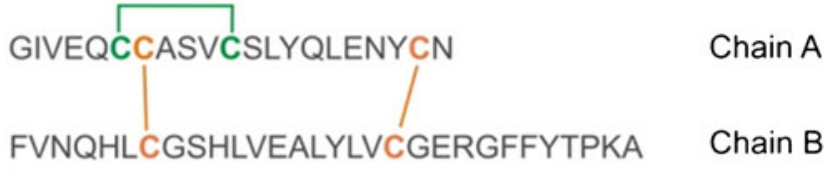

$\alpha$ - Lactalbumin
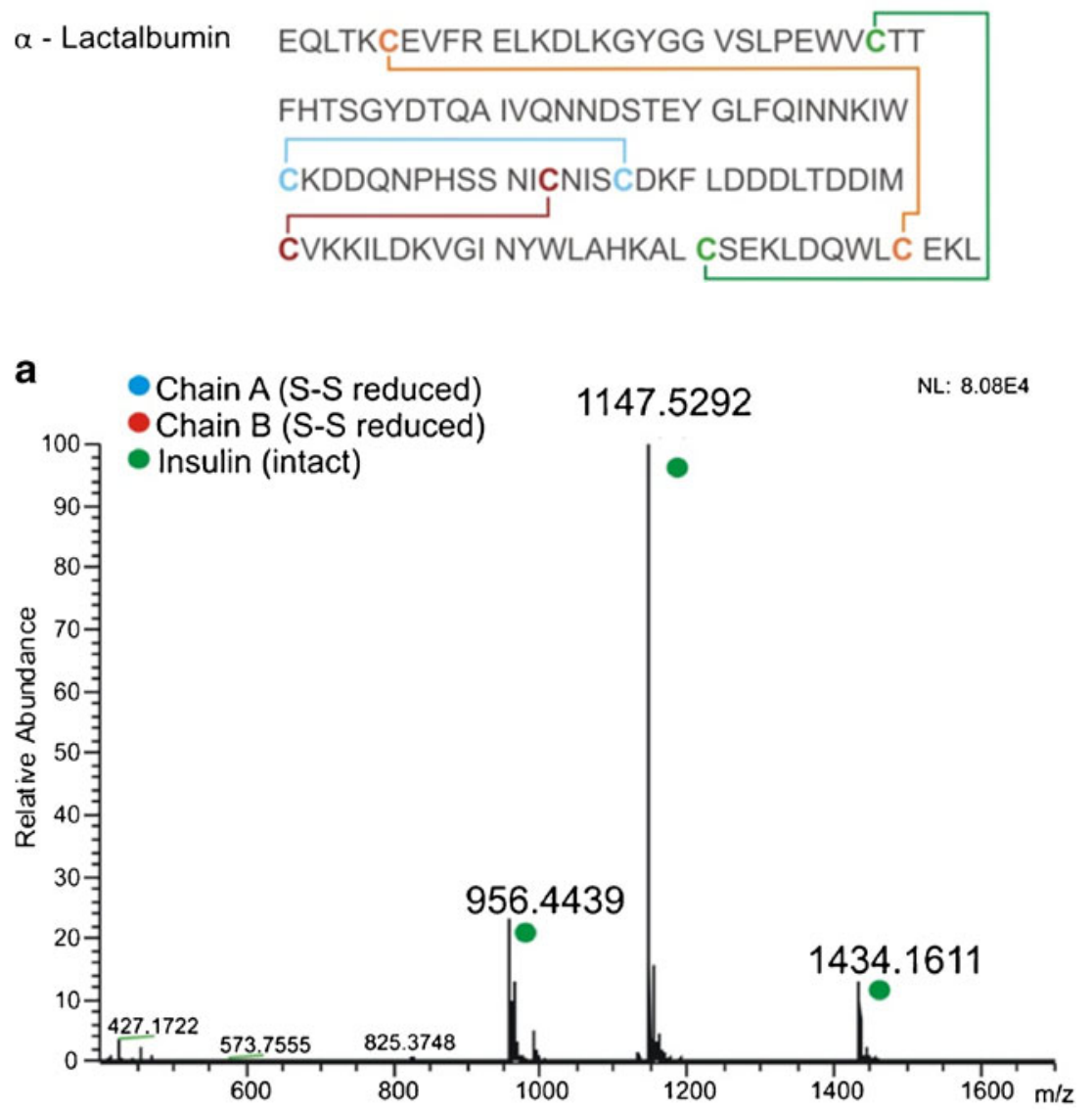

b

NL: $1.95 E 4$

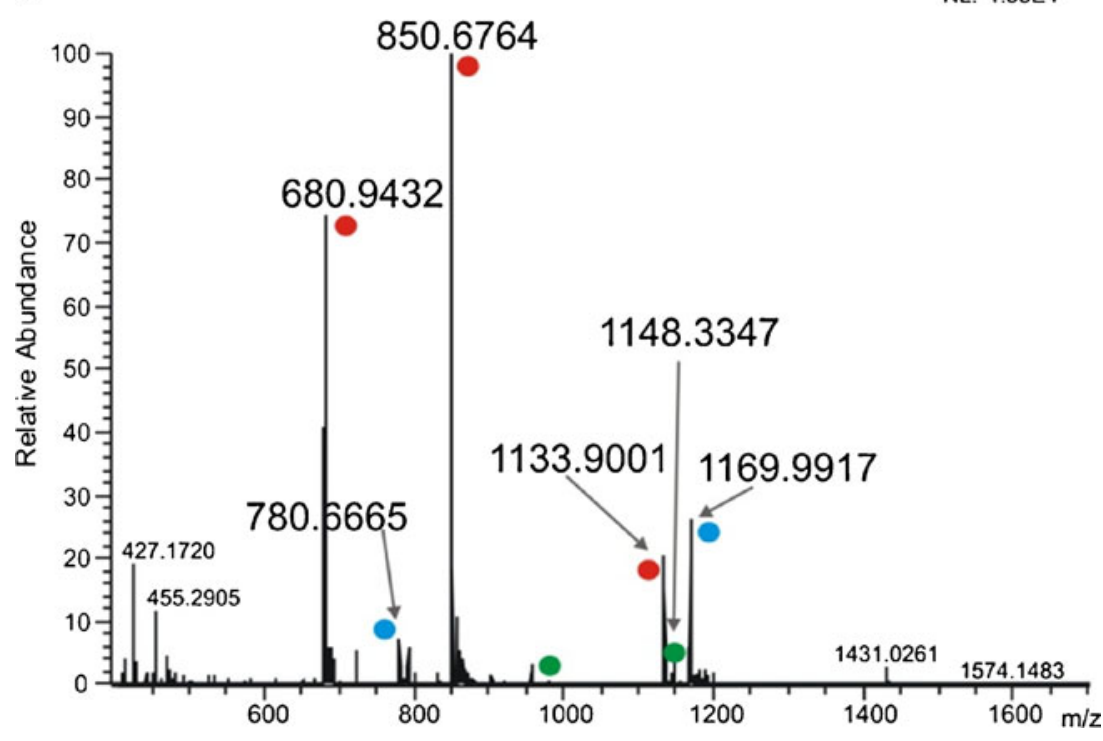




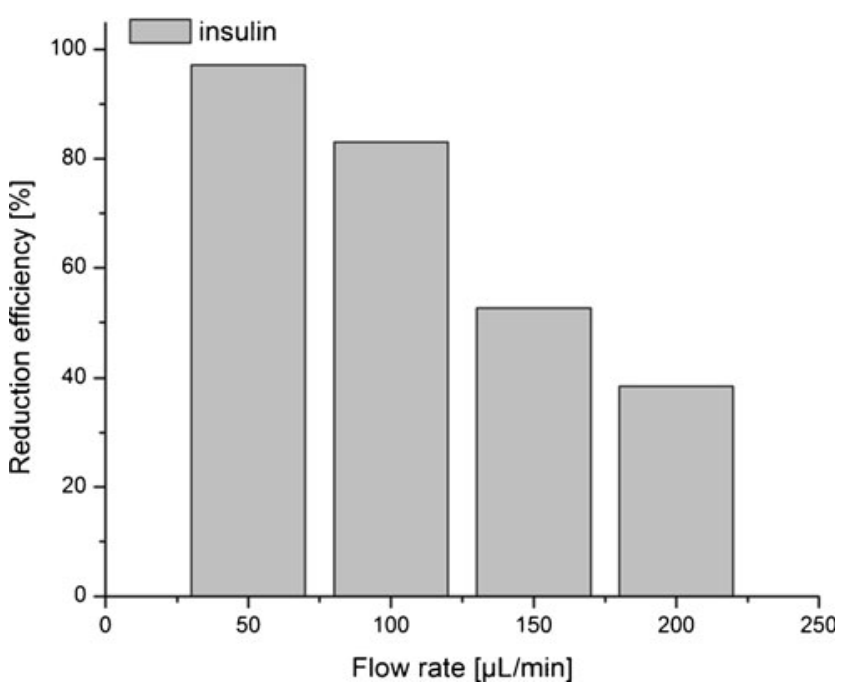

Fig. 5 Reduction efficiency of insulin as a function of flow rate
4 and 17) and is therefore difficult to detect in positive ion mode [31]. However, two low abundant peaks at $\mathrm{m} / \mathrm{z} 1,169$ and 780 were observed in the mass spectrum and are in agreement with theoretical masses of reduced $[\mathrm{M}+2 \mathrm{H}]^{2+}$ and $[\mathrm{M}+3 \mathrm{H}]^{3+}$ ions of chain A (Fig. 4b). Moreover, the isotopic pattern for the [M+ $3 \mathrm{H}]^{3+}$ ion shows that the intra-chain disulfide bond is near $100 \%$ reduced, and only low abundant ions of the species with the intra-bond present are observed (Fig. S2, ESM). Based on the reduction of the abundances of insulin ions, a reduction efficiency of $>98 \%$ was determined.

For efficient disulfide bond reduction proper selection of experimental conditions is essential. During method development, the electrode material, the square wave potential settings, the solution composition and the flow rate were identified as parameters influencing the reduction efficiency observed for insulin. a

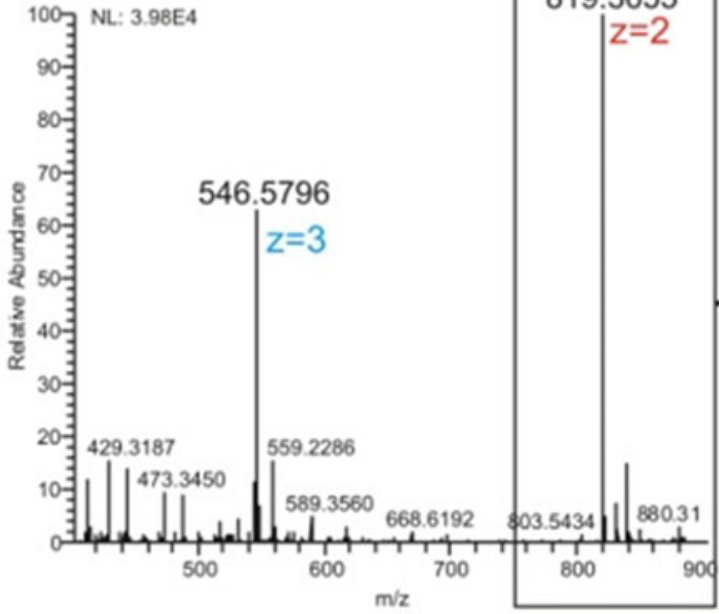

b

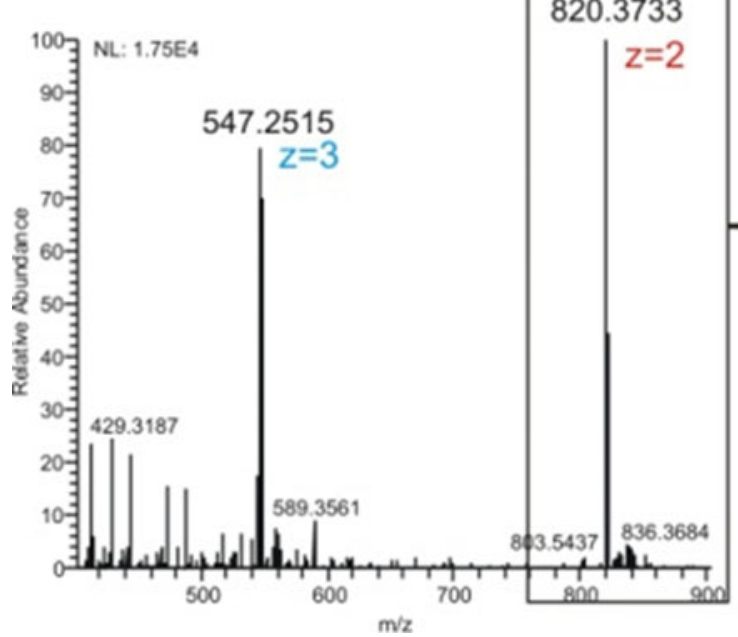

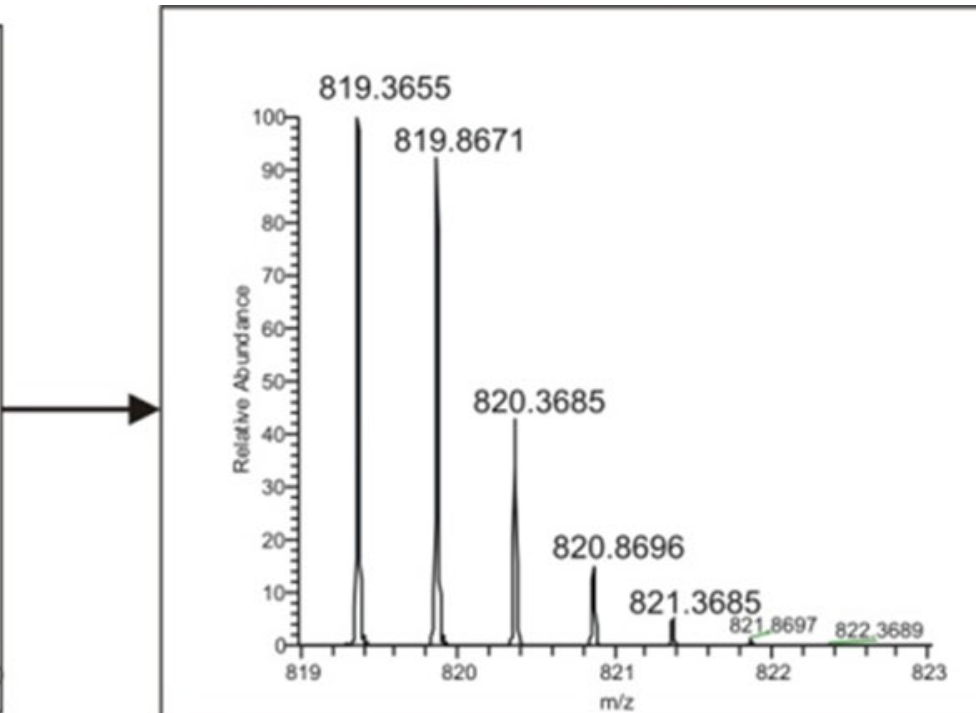
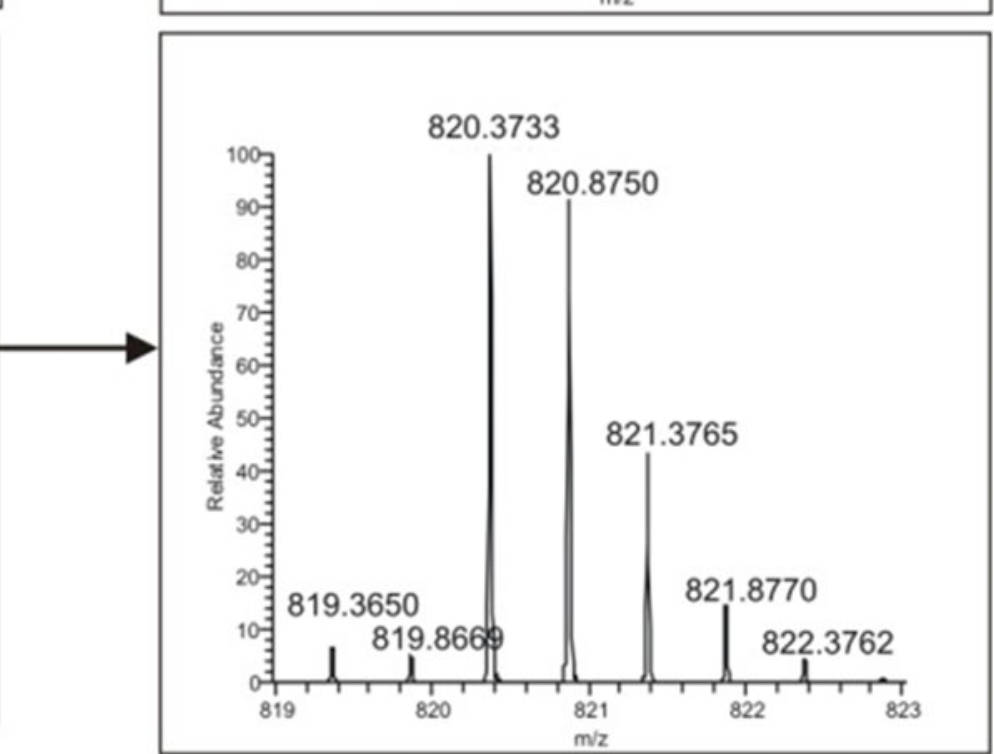

Fig. 6 Mass spectra of $3 \mu \mathrm{mol} / \mathrm{L}$ somatostatin in $1 \%$ formic acid and $10 \% \mathrm{ACN}$ with reactor cell OFF (a) and ON (b) and a close up of the isotopic distribution of the $[\mathrm{M}+2 \mathrm{H}]^{2+}$ ions. Pulse settings are the same as in Fig. 2 
a

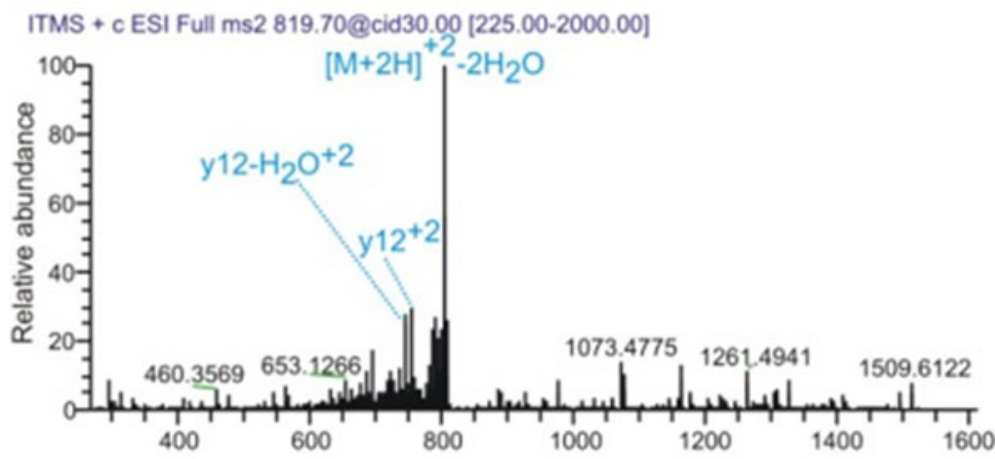

b

ITMS + p ESI Full ms2 820.00@ @id30.00 [225.00-2000.00]
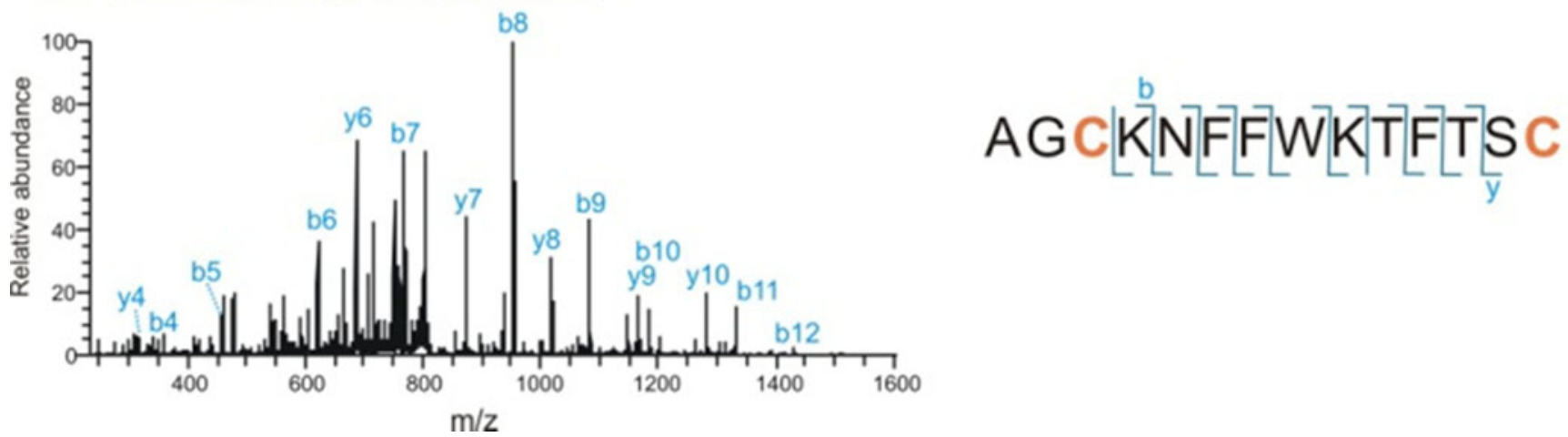

Fig. 7 CID fragmentation of $3 \mu \mathrm{mol} / \mathrm{L}$ somatostatin in $1 \%$ formic acid and $10 \% \mathrm{ACN}$ with the EC reactor cell OFF (a) and ON (b). The sequence of somatostatin with indicated backbone cleavages is shown. Pulse settings are the same as in Fig. 2. Mass spectra were recorded with a LTQ analyzer

A Ti-based electrode in combination with square-wave pulses was found to provide constantly high reduction efficiency. Mechanistic details of electron transfer reactions (redox reactions) at oxide-covered metal electrodes are well understood [32, 33]. Generally, metal electrodes need a distinct thin layer of metal oxide to catalyze the redox reaction; however, increasing thickness of the oxide layer may have a negative effect on the electron transfer rate [34]. To avoid electrode fouling, a square-wave pulse with an alternating positive and negative potential was developed (Fig. 2). During the oxidative potential, a metal oxide layer is formed on the WE, which is subsequently removed during the reductive potential of the square wave. As a result, the oxide layer does not accumulate and the surface of the working electrode remains active.

The importance of alternating the polarity of the potential is illustrated by changing the positive potential of E2 to a negative potential, which resulted in a significantly lower reduction yield as shown in Fig. S3 (ESM). Subsequently, at constant $\mathrm{E} 2$ of $1 \mathrm{~V}$ the $\mathrm{E} 1$ potential values between $-0.5 \mathrm{~V}$ and $-3 \mathrm{~V}$ were tested showing different reduction efficiency. Figure S4 (ESM) shows comparison between $\mathrm{E} 1=-0.7 \mathrm{~V}$ and $\mathrm{E} 1=-1.5 \mathrm{~V}$. A more negative $\mathrm{E} 1$ potential results in more efficient reduction.

\section{AGC $\underset{y}{\operatorname{CoN}}$ KNFWKTFTSC}

Also the duration, $\mathrm{t} 1$ and $\mathrm{t} 2$ of the potential steps can be used to achieve partial or full disulfide reduction. Typically, E1 was applied for 2,000 ms and E2 for 1,000 ms, and conversion of intact insulin was approximately $98 \%$ (Fig. 4a, b). Shortening t 1 and t 2 to $200 \mathrm{~ms}$ and $100 \mathrm{~ms}$, respectively, resulted in lower conversion of insulin of about $50 \%$ (data not shown here). With this example, we demonstrate a convenient way to modulate the reduction efficiency by tuning the square-wave pulse parameters E1, E2 and the time intervals. This is particularly useful for assignment of disulfide bonds in proteins, when controlled partial reduction is desired, rather than $100 \%$ reduction.

Electrochemical reduction was found to work well in aqueous solutions containing formic acid and acetonitrile as modifiers. These compounds are commonly used mobile phase additives in LC/MS of proteins and peptides. In comparison to

Fig. 8 Mass spectra of $5 \mu \mathrm{mol} / \mathrm{L} \alpha$-lactalbumin in $1 \%$ formic acid and $50 \%$ acetonitrile with the EC reactor cell OFF (top) and ON with different E1 potentials as noted in the figure. The E2 potential was $1,000 \mathrm{mV}$, t 1 and $\mathrm{t} 2$ was set to 1,990 and $1,010 \mathrm{~ms}$, respectively (for pulse settings see also Fig. 2). The charge state corresponding to the native protein (with the reactor OFF) is labeled in blue. The multiply charged ions appearing as a result of the electrochemical reaction have the charge state indicated in red 

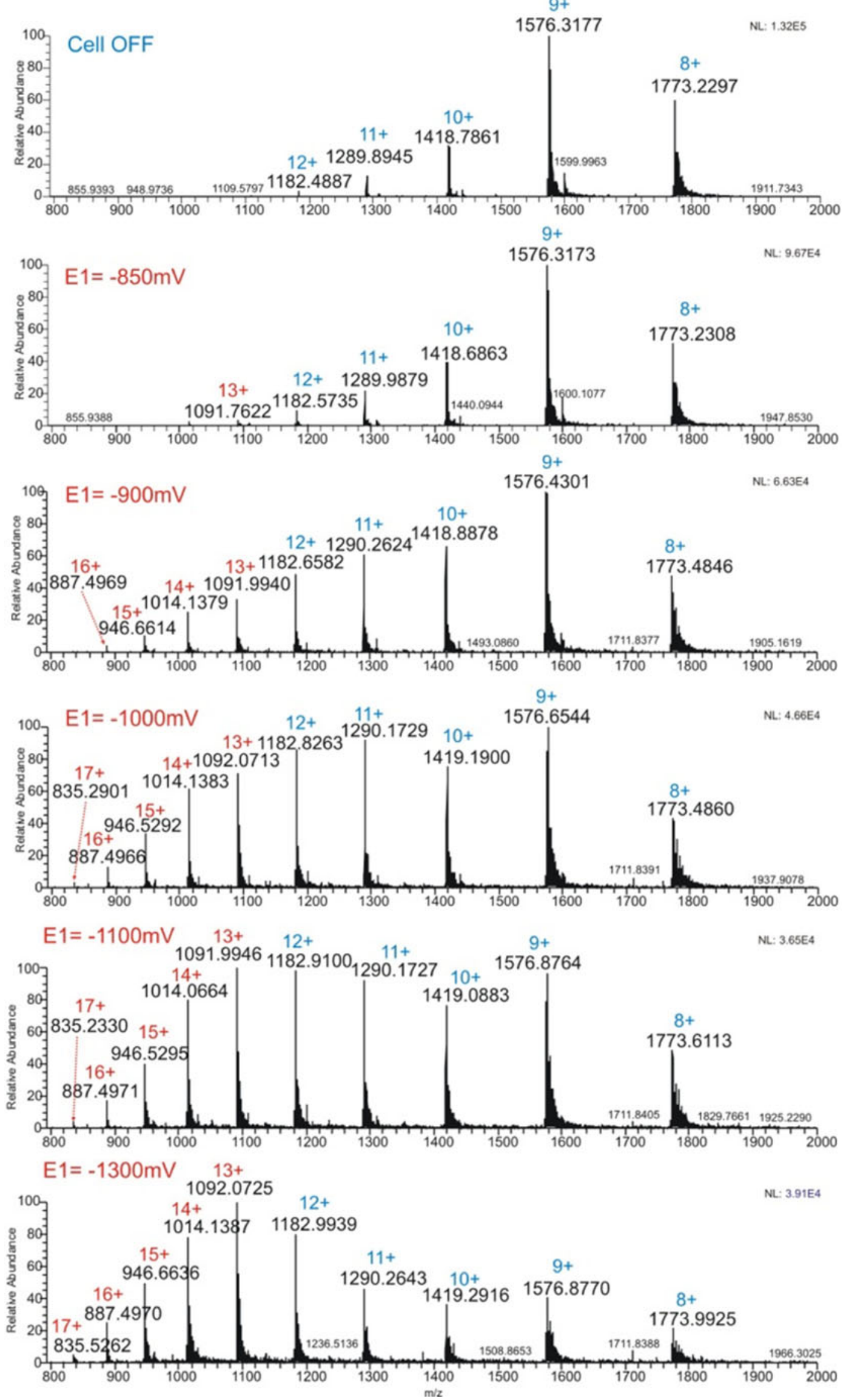
standard LC/MS conditions, however, $0.5-1 \%$ formic acid should be added to the sample solution to ensure efficient reduction. Formic acid is used as supporting electrolyte and can enhance the electron exchange. It can be considered as an electron donor and used as reducing agent [35]. With respect to the acetonitrile content, the reduction efficiency was only slightly decreased (to $87 \%$ based on the decrease in abundance of the +5 -fold protonated intact insulin ion) by increasing the concentration of acetonitrile to $50 \%$ comparing to the typically used $10 \%$ acetonitrile solution.

The impact of the flow rate on performance is shown in Fig. 5 (for more information see also Fig. S5 in ESM). At $50 \mu \mathrm{l} / \mathrm{min}$, almost complete reduction of insulin was achieved. By increasing the flow rate, a decrease of insulin cleavage was observed. This observation clearly suggests that the flow rate can be used to control the reduction efficiency. Thus, by proper setting of the flow rate it should become possible to switch between complete and partial disulfide bond reduction.
Partial reduction could be of particular importance to localize disulfide bonds and to study the impact of individual disulfide bonds on peptide and protein structures (see below).

\section{EC/MS of somatostatin}

Somatostatin was selected as target to demonstrate the usefulness of EC/MS for studying peptides containing an internal disulfide bond (Fig. 3). Somatostatin (also called somatropin) is a peptide hormone. There are two active forms; the peptide containing 14 amino acids and an internal disulfide bridge was used for this study. The mass spectrum of the native form of somatostatin is shown in Fig. 6. The monoisotopic peak of the $[\mathrm{M}+2 \mathrm{H}]^{2+}$ ion was detected at $819.3655 \mathrm{~m} / z$. After electrochemical reduction, the $\mathrm{m} / \mathrm{z}$ values were shifted to 820.3733 indicating the cleavage of the disulfide bond (Fig. 6). Based on the reduction of the intensity of $819.3655 \mathrm{~m} / \mathrm{z}$, a reduction efficiency of $>90 \%$ was determined.
Fig. 9 Zoom of the overlapping isotopic pattern of the +9 ion of $\alpha$ lactalbumin measured with the EC reactor cell turned OFF (top), and $\mathrm{ON}$ at $\mathrm{E} 1=-1,000 \mathrm{mV}$ and $\mathrm{E} 1=-1,300 \mathrm{mV}$. E2 was $1,000 \mathrm{mV}$ in both cases. See also Fig. 2 for pulse settings. The protein was dissolved in $1 \%$ formic acid and $50 \%$ acetonitrile. The bottom panel shows the isotope simulation for $\alpha$ lactalbumin with all four bonds reduced. Simulation was done using Xcalibur software. The highest peak in each spectrum is labeled in bold

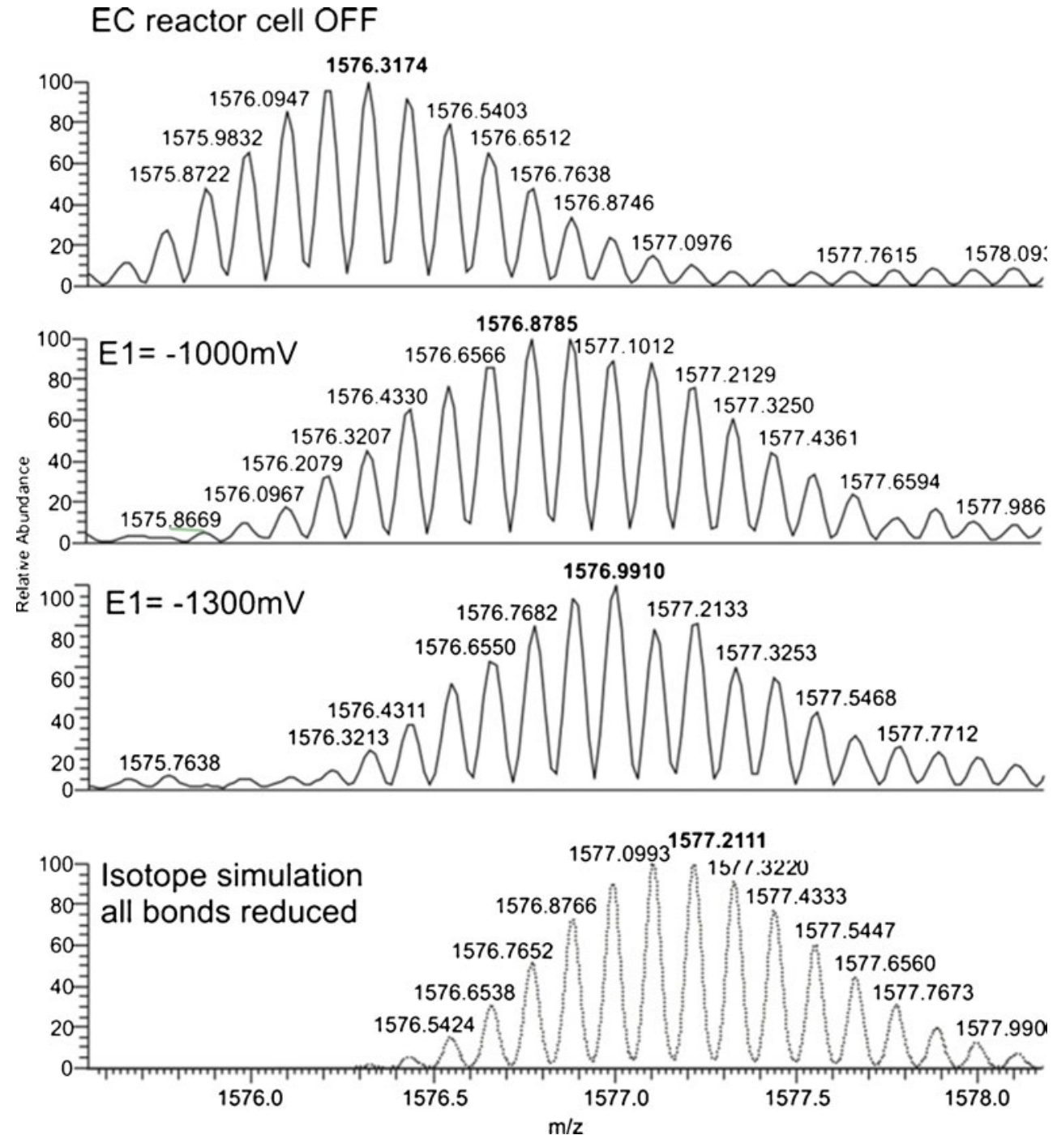


Disulfide bond cleavage can be of particular importance for obtaining high sequence coverage in MS/MS experiments. This is demonstrated by CID of the native and the reduced forms of somatostatin (Fig. 7). In the CID spectrum of the native somatostatin, only one abundant fragment ion was detected. This ion was identified as the y12-ion produced by cleavage of the first two amino acids (outside the loop formed by the disulfide linkage). After electrochemical reduction of the peptide, almost full series of $y$ - and b-type ions were detected which enabled unequivocal peptide identification via de novo sequencing and library search.

\section{EC/MS of $\alpha$-lactalbumin}

$\alpha$-Lactalbumin was chosen to demonstrate the usefulness of EC/MS for studying proteins containing multiple internal disulfide bonds. $\alpha$-Lactalbumin contains 123 amino acids. The globular structure of $\alpha$-lactalbumin is stabilized by four disulfide bonds: Cys6-Cys120, Cys28-Cys111, Cys61-Cys77 and Cys73-Cys91 (Fig. 3). The mass spectrum of the native protein is shown in Fig. 8. The charge states observed ranged from +8 to +12 ; the most abundant ion was the nine times positively charged ion. The electrochemical reduction caused a shift of the charge state distribution to more highly charged ions (Fig. 8). In the fully reduced form, the $[\mathrm{M}+13 \mathrm{H}]^{13+}$ ion showed the highest signal abundance. Obviously, reduction of disulfide bonds facilitated unfolding of the protein, which enabled accumulation of a larger number of charges on the surface of the protein. This phenomenon has also been described in literature [36, 37] and was confirmed by Zhang et al. [28] recently. Furthermore, the reducing potential was used as a means to control the extent of disulfide bond cleavage. More negative potentials gave rise to an increased shift of the charge state distribution indicating increased disulfide bond cleavage and unfolding of the protein. This partial opening might find immediate application in top-down proteomics for the location of disulfide bonds as well as to study their individual accessibility. Figure 9 shows a close up of the $[\mathrm{M}+9 \mathrm{H}]^{9+}$ ion of $\alpha$-lactalbumin measured with the $\mathrm{EC}$ reactor cell off (no reduction) and on with two different E1 potentials of $-1,000$ and $-1,300 \mathrm{mV}$. The overlapping isotopic patterns correspond to different reduction states of the protein including also $\alpha$-lactalbumin with all bonds reduced.

\section{Conclusions}

We have shown the successful use of EC reactor cell with a titanium-based working electrode for reduction of disulfide bonds in proteins and peptides. The method is fast, efficient and robust. Due to use of volatile solvent additives (i.e. formic acid and acetonitrile), the electrochemical reduction can be hyphenated online to MS. Furthermore, the reduction efficiency is controllable by tailoring experimental conditions, such as the flow rate and the square wave pulse settings. This enables to easily switch between complete and partial reduction. Complete reduction was found to be beneficial to increase the sequence coverage in CID. Partial reduction might enable the location of disulfide bonds within proteins and peptides as well as studies on their accessibility.

The electrochemical reduction shows great potential in proteomics applications particularly for the assignment of disulfide bonds in proteins and the cleavage of TCEPresistant disulfide bonds is important for hydrogen-deuterium (H-D) exchange.

To obtain detailed information on the assignment of disulfide bonds in a protein, a $100 \%$ reduction of all bonds is not always desirable. The use of a chemical reductant leads usually to $100 \%$ reduction, and the information about the assignment of disulfide bonds is lost. The electrochemical reduction can be a very good alternative for disulfide bond assignment in proteins. Simply by turning on the EC reactor cell and changing the settings, partial to full reduction can be achieved. The reduction rate can be controlled in several ways, e.g. by adjusting the square-wave pulse, flow rate, temperature, mobile phase or cell volume (spacer) giving new opportunities for disulfide bonds assignment studies. Recently, Zheng et al. reported selective disulfide bond reduction at different potentials using an online EC reactor cell, a conductive diamond working electrode and a pepsin column for digestion [29].

Disulfide bond reduction using an EC reactor cell is of particular interest for $\mathrm{H}-\mathrm{D}$ exchange experiments. H-D exchange is used to analyse the tertiary structure of proteins, by exposing them in their natural folded state to deuterons. Regions of a protein that are easily accessible by the solvent will exchange rapidly, folded regions hardly exchange. For reliable results the back-exchange must be minimized (quenched), and after disulfide bond reduction and protein proteolysis the peptide fragments must be analysed as quickly as possible [38]. Traditionally the disulfide bond reduction is performed under quench conditions ( $\mathrm{pH} 2.5$ and $0{ }^{\circ} \mathrm{C}$ ) using a chemical reductant such as TCEP. However, under these conditions TCEP has limited efficiency [39]. The electrochemical reduction using an EC reactor cell is efficient under quench conditions, and furthermore, the residence time in the EC reactor cell to obtain full reduction is only $10-15 \mathrm{~s}$. The application of $\mathrm{EC}$ reduction for $\mathrm{H}-\mathrm{D}$ exchange in proteins was recently presented by Mysling and co-workers during ASMS 2013 conference in the oral presentation entitled "Electrochemical reduction of disulfide bonds for use in protein hydrogen/ deuterium exchange monitored by mass spectrometry". Future investigations will be focused on LC/EC/MS method for electrochemical reduction of proteins and peptides including reduction of disulfide bonds in the high molecular weight proteins as antibodies. 
Acknowledgments The authors would like to acknowledge the division of Analytical BioSciences (Leiden University, The Netherlands) for access to their MS facility and to thank Prof. Dr. Herbert Oberacher (Institute of Legal Medicine, Innsbruck Medical University, Austria) for his valuable discussions during the preparation of this manuscript.

Open Access This article is distributed under the terms of the Creative Commons Attribution License which permits any use, distribution, and reproduction in any medium, provided the original author(s) and the source are credited.

\section{References}

1. Hogg PJ (2003) Trends Biochem Sci 28:210-214

2. Aebersold R, Mann M (2003) Nature 422:198-207

3. Domon B, Aebersold R (2006) Science 312:212-217

4. Armirotti A, Damonte G (2010) Proteomics 10:3566-3576

5. Mormann M, Ebne J, Schwöppe C, Mesters RM, Berdel WE, PeterKatalinić J, Pohlentz G (2008) Anal Bioanal Chem 392:831-838

6. Gorman J, Wallis TP, Pitt JJ (2002) Mass Spectrom Rev 21:183-216

7. Zubarev RA, Kelleher NL, McLafferty FW (1998) J Am Chem Soc 13:3265-3266

8. Syka JEP, Coon JJ, Schroeder MJ, Shabanowitz J, Hunt DF (2004) Proc Natl Acad Sci U S A 101:9528-9533

9. Zubarev RA, Kruger NA, Fridriksson EK, Lewis MA, Horn DM, Carpenter BK, McLafferty FW (1999) J Am Chem Soc 121:28572862

10. Cole SR, Ma X, Zhang X, Xia Y (2012) J Am Soc Mass Spectr 23: 310-320

11. Zahler WL, Cleland WW (1964) Biochemistry 3:480-482

12. Getz EB, Xiao M, Chakrabarty T, Cooke R, Selvin PR (1999) Anal Biochem 273:73-80

13. Jahn S, Karst U (2012) J Chromatogr A 1259:16-49

14. Chen L, Hofmann D, Klumpp E, Xiang X, Chen Y, Stephan Küppers S (2012) Chemosphere 89:1376-1383
15. Erb R, Plattner S, Pitterl F, Brouwer HJ, Oberacher H (2012) Electrophoresis 33:614-621

16. Nouri-Nigjeh E, Permentier HP, Bischoff R, Bruins AP (2011) Anal Chem 83:5519-5525

17. Permentier HP, Bruins AP, Bischoff R (2008) Mini Rev Med Chem 8: $46-56$

18. Roeser J, Alting NFA, Permentier HP, Bruins AP, Bischoff R (2013) Anal Chem 85:6626-6632

19. Cecil R, Weitzman PDJ (1964) Biochem J 93:1-11

20. Honeychurch MJ (1997) Bioelectroch Bioener 44:13-21

21. Kuznetsov BA, Shumakovich GP, Mestechkina NM (1988) J Electroanal Chem 248:387-398

22. Garvie CT, Straub KM, Lynn RK (1987) J Chromatogr 413:43-52

23. Sun Y, Andrews PC, Smith DL (1990) J Protein Chem 9:151-157

24. Sun Y, Smith DL, Shoupt RE (1991) Anal Biochem 197:69-76

25. Qiao L, Bi H, Busnel J-M, Liuand B, Girault HH (2008) Chem Commun 47:6357-6359

26. Zhang Y, Dewald HD, Chen H (2011) J Proteome Res 10:1293-1304

27. Lu M, Wolff C, Cui W, Chen H (2012) Anal Bioanal Chem 403:345355

28. Zhang Y, Cui W, Zhang H, Dewald HD, Chen H (2012) Anal Chem 84:3838-3842

29. Zheng Q, Zhang H, Chen H (2013) Int J Mass Spectrom. doi:10. 1016/j.ijms.2013.04.009

30. Kraj AU, Brouwer HJ, Chervet JP (2012) US13/608907

31. Loo JA, Ogorzalek Loo RR, Light KJ, Edmonds CG, Smith RD (1992) Anal Chem 64:81-88

32. Lyons MEG, Brandon MP (2010) J Electroanal Chem 641:119-130

33. Stitz A, Buchberger W (1994) Electroanal 6:251-258

34. Boukhalfa S, Evanoff K, Yushin G (2012) Energy Environ Sci 5: 6872-6879

35. Garron A, Epron F (2005) Water Res 39:3073-3308

36. Loo JA, Edmonds CG, Udseth HR, Smith RD (1990) Anal Chem 62: 693-698

37. Katta V, Brian T, Chait BT (1991) J Am Chem Soc 113:8534-8535

38. Wales TE, Engen JR (2006) Mass Spectrom Rev 25:158-170

39. Cline DJ, Thorpe C (2004) Biochemistry 43:15195-15203 